\title{
Independent Components of Magnetoencephalography: Single-Trial Response Onset Times
}

\author{
Akaysha C. Tang, ${ }^{*}+\ddagger^{\prime 1}{ }^{1}$ Barak A. Pearlmutter, $\dagger^{\prime} \ddagger$ Natalie A. Malaszenko,* and Dan B. Phung* \\ *Department of Psychology, †Department of Neurosciences, and $¥$ Department of Computer Science, University of New Mexico, \\ Albuquerque, New Mexico 87131
}

Received March 27, 2002

\begin{abstract}
We recently demonstrated that second-order blind identification (SOBI), an independent component analysis (ICA) method, can separate the mixture of neuronal and noise signals in magnetoencephalographic (ME G) data into neuroanatomically and neurophysiologically meaningful components. When the neuronal signals had relatively higher trial-to-trial variability, SOBI offered a particular advantage in identifying and localizing neuronal source activations with increased source detectability (A. C. Tang et al., 2002, Neural Comput. 14, 1827-1858). Here, we explore the utility of SOBI in the analysis of temporal aspects of neuromagnetic signals from MEG data. From SOBI components, we were able to measure single-trial response onset times of neuronal populations in visual, auditory, and somatosensory modalities during cognitive and sensory activation tasks, with a detection rate as high as $\mathbf{9 6 \%}$ under optimal conditions. Comparing the SOBI-aided detection results with those obtained directly from the sensors, we found that with SOBI preprocessing, we were able to measure, among a greater proportion of trials, single-trial response onset times that are above background neuronal activity. We suggest that SOBI ICA can improve our current capability in measuring single-trial responses from human subjects using the noninvasive brain imaging method ME G. 2002 Elsevier Science (USA)
\end{abstract}

\section{INTRODUCTION}

Second-order blind identification (SOBI) (Belouchrani et al., 1993; Cardosa, 1994) is one of the independent component analysis (ICA) algorithms that take mixtures of signals from multiple sources as input and decompose the mixtures into components. It has recently been applied to the analysis

\footnotetext{
${ }^{1}$ To whom correspondence should be addressed at the Department of Psychology, Logan Hall, University of New Mexico, Albuquerque, NM 87131. Fax: (505) 277-1394. E-mail: akaysha@unm.edu.
}

of magnetoncephalographic (MEG) data to decompose the mixture of magnetic sensor signals into various neuronal and noise components (Tang et al., 2000a,Tang et al., 2002; Ziehe et al., 2000; Wübbeler et al., 2000; M ackert et al., 2001). As do other ICA algorithms (Vigário et al., 1998), SOBI ICA can effectively isolate signals associated with ocular artifact, 60- $\mathrm{Hz}$ noise, and other noise sources, such as slow DC drift and large sensor quantum state changes (Tang et al., 2000a, 2002). Thus, SOBI can be viewed as a preprocessing tool that cleans up the raw sensor data to all ow researchers to focus on neuronal signals of interest (Tang et al., 2002). In addition, SOBI ICA, as well as fICA (Hyvärinen and Oja, 1997), generates components that can be further localized with the equival ent current dipole (ECD) modeling method (Vigário et al. 1999; Tang et al., 2002). Most importantly, SOBI uses detailed temporal information present in the nonaveraged continuous sensor data to generate components that correspond to activation of separate brain regions predicted from the tasks (Mackert et al., 2001; Tang et al., 2002). By virtue of separating various neuronal and noise sources into separate components, SOBI offers a way of improving the effective signal-to-noise $(\mathrm{S} / \mathrm{N})$ ratios for activation of each different brain region.

Recently, we examined whether SOBI can lead to increased probability of detecting and localizing sources of neuronal activation (Tang et al., 2002). Because SOBI can isolate various noise signals from neuronal signals, it was hypothe sized that SOBI should be most effective in improving neuromagnetic source localization when the data are noisy. We applied SOBI to MEG data collected from multiple subjects performing multiple cognitive tasks characterized by relatively large trial-to-trial variability (thus noisier data). These tasks offered particularly challenging data sets for testing whether SOBI provided any advantage in neuromagnetic source localization.

This study yielded three major results (Tang et al., 2002). First, SOBI decomposed the sensor signals into components whose time courses and sensor projections corresponded to neurophysiologically and neuroanatomically meaningful activations of separate brain regions. The locations of source activation generated from the components' sensor projections 


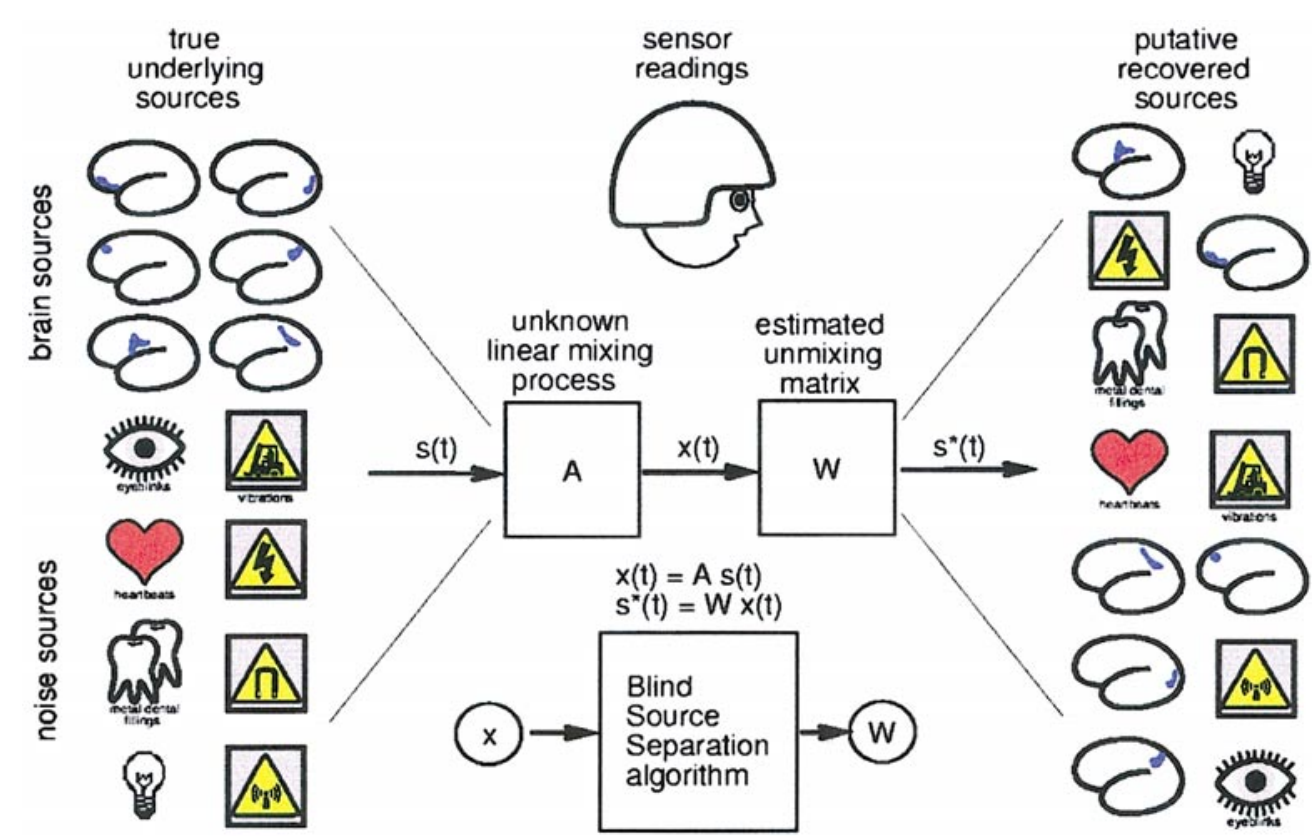

FIG. 1. The ICA process. Signals from the brain and other noise sources $\mathbf{s}(\mathrm{t})$ are mixed through an unknown linear mixing process $\mathbf{A}$, resulting in the sensor readings $\mathbf{x}(\mathrm{t})=\mathbf{A} \mathbf{s}(\mathrm{t})$. ICA finds an unmixing matrix $\mathbf{W}$ that maps from the sensor signals to recovered components $\hat{\mathbf{s}}(\mathrm{t})=\mathbf{W} \mathbf{x}(\mathrm{t})$. The entries of the attenuation matrix $\mathbf{A}=\mathbf{W}^{-1}$ describe how strongly each sensor responds to each component.

were highly reproducible across multiple tasks and the SOBI components' temporal profiles showed task modulation. The source localization was also highly reproducible across subjects when presented with similar sensory stimulation.

Second, with the aid of SOBI, task activation of expected brain regions can be detected with significantly increased probability when the stimuli that triggered the activation were highly variable. For example, left and right somatosensory activation due to incidental stimulation of right and left thumbs as a result of button presses can be identified and localized unambiguously from SOBI components in over $90 \%$ of the cases. In contrast, an attempt to localize the same somatosensory source activation from the sensor signals directly failed in more than $50 \%$ of cases. When $\mathrm{S} / \mathrm{N}$ ratios were high, localization of neuromagnetic source activation directly from the sensor was as successful as using SOBI. For example, when a high-contrast suprathreshold visual stimulus was presented, activation of early visual processing areas was easily detected directly from the sensor signals.

Third, because SOBI components' sensor projections are fixed in time, or time-invariant, when localizing this component, there was no need to make subjective decisions with regard to which point in time to fit a dipole. In conventional approaches, one must choose some point in time, typically the peak of the evoked response potentials (ERPs) from a sensor with the largest peak from a particular region of interest (ROI ), in order to fit the sensor projection to a source model. Implicitly, one makes the subjective judgment of at what point in time sensor signals contain mostly the signal from a particular underlying source of interest. With the time-invariance of SOBI components' sensor projections, this subjective step can be removed with the aid of SOBI .
Finally, when focal activation was expected from the tasks, sensor projections of SOBI components had focal spatial patterns of activation. For early sensory processing, these focal sensor projections were almost invariantly dipolar or multidipolar in their magnetic field patterns. This eliminates the need to subjectively determine which sensors to exclude near the border of the ROI in an attempt to make the field dipolar prior to single ECD modeling. Reducing such subjective decisions can help to improve the reproducibility of source localization results. ${ }^{2}$

In the present study, we continued to explore SOBI's utility in the analysis of temporal aspects of brain neuromagnetic responses. Specifically, we investigated whether one can measure single-trial response onset times from those SOBI components that correspond to nonaveraged continuous activation of particular brain regions. Because SOBI increases the effective $S / N$ ratio by separating the noise from the neuromagnetic signals and by separating various neuronal source signals from each other, we expect SOBI to improve single-trial response measurement over the measurement made directly on the sensor signals that contain a mixture of signals from noise and the neuronal signals of interest.

\section{METHODS}

We collected MEG data during a simple sensory activation task and four cognitive tasks. These tasks offered data col-

\footnotetext{
${ }^{2}$ For SOBI components that correspond to distributed neuromagnetic activation, magnetic field tomography (MFT) or other distributed source modeling methods should be used. So far, the SOBIMFT combination has not been explored.
} 
(a)

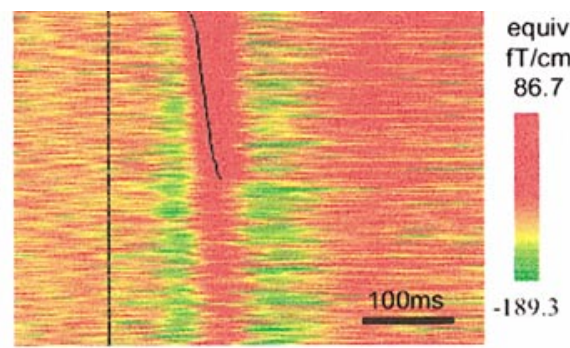

(c)

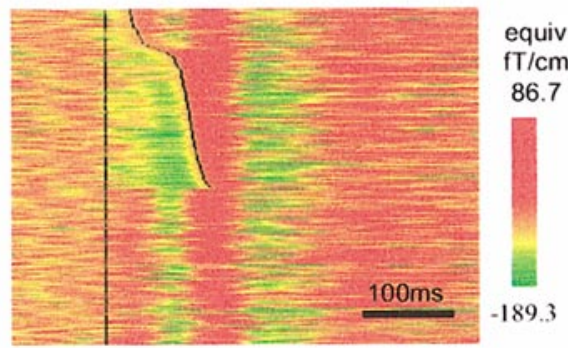

(e)

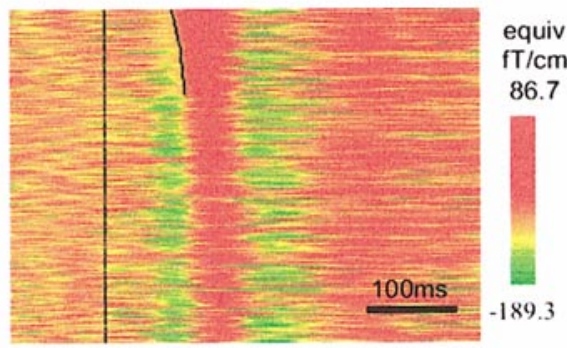

(g)

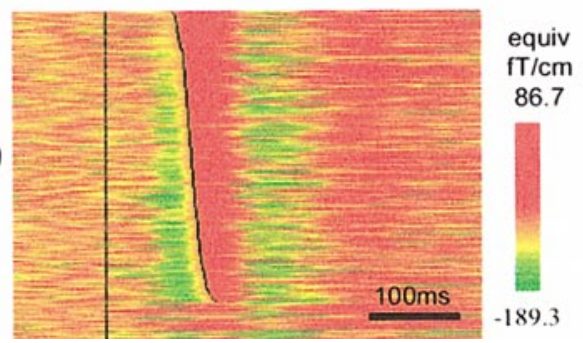

(b)

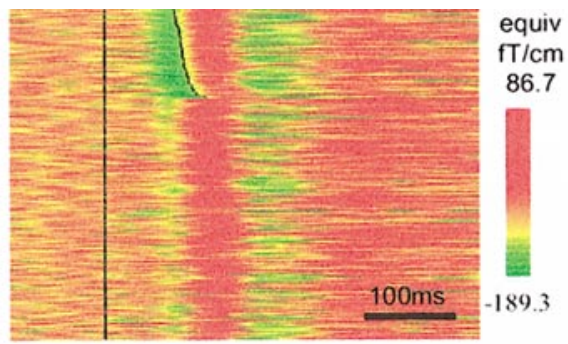

(d)

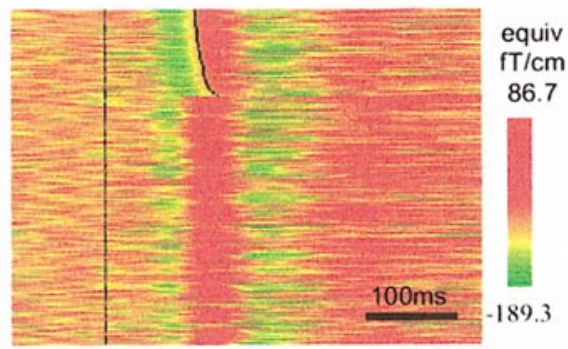

(f)

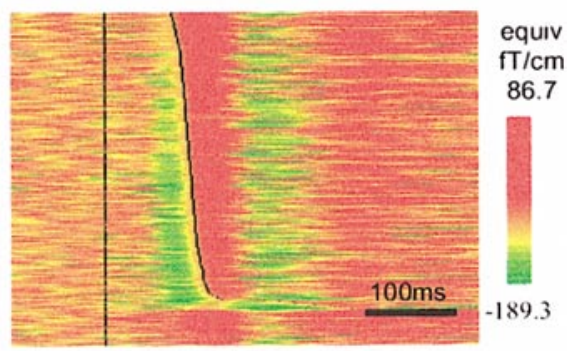

(h)

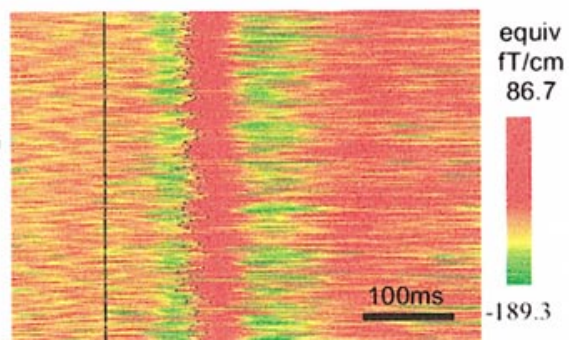

FIG. 2. (a-g) Examples of suboptimal singletrial onset detection from a single SOBI component. (a) Too high a threshold; (b) too low a threshold; (c) too early a beginning window (WB); (d) too late a WB; (e) too early an end window (WE); (f) too late a WE; (g) an optimal detection; trials were sorted in order of increasing onset times. (h) An example of optimal detection shown in natural trial order. See Table 1 for associated detection parameters and results.

lected at both high and low $\mathrm{S} / \mathrm{N}$ ratios. These data involve activation of neuronal sources from three major sensory modalities. The largest variability in neuronal source activation was induced by highly variable incidental somatosensory stimulation due to button-press responses. The second largest variability in neuronal source activation was induced by presentation of visual stimuli without the use of a fixation point. The smallest variability in neuronal source activation was induced by a binaural stimulation from a pure tone. The number of stimulations per modality varied between 90 and 270 trials. Because the tasks were originally designed for purposes other than testing the SOBI algorithm, detailed descriptions of the tasks (Tang et al., 2002) are omitted here.

\section{Inputs and 0 utputs}

The inputs to SOBI are time-varying vectors $\mathbf{x}(\mathrm{t})$ containing the continuous (i.e., not averaged) MEG time series from all 122 sensors for the entire period of the experiment, sampled at 300 and $600 \mathrm{~Hz}$ and bandpass filtered at $0.03-100 \mathrm{~Hz}$. The output of the algorithm is a $122 \times 122$ matrix, $\mathbf{W}$, which maps from the vector of sensor values $\mathbf{x}(\mathrm{t})$ to the vector of 
recovered component values $\hat{\mathbf{s}}(\mathrm{t})=\mathbf{W} \mathbf{x}(\mathrm{t})$ (Fig. 1 ), up to a scaling and permutation of the components. The scale factor was chosen to give the recovered components sensible units (effective fT/cm), as described by Tang et al. (2002, Appendix B.3). U sing the matrix $\mathbf{W}$, one maps the input sensor signals $\mathbf{x}(\mathrm{t})$ to the recovered component signals $\hat{\mathbf{s}}(\mathrm{t})$.

SOBI generated 122 components, ${ }^{3}$ each a one-dimensional time series with an associated field map (Tang et al., 2002). Each component potentially corresponds to a set of magnetic field generators. These generators include various neuromagnetic sources associated with neural processing taking place within the brain and noise sources from elsewhere. Taskrelated component activation that corresponds to neuromagnetic source activation of different brain regions can be identified by examining the stimulus- or response-triggered averages. Typically, of all 122 components, only a small number of components showed task-related activation. The components with both clear ERPs and anatomically meaningful sensor projections ${ }^{4}$ are further analyzed.

\section{Temporal Aspects of SOBI Components}

The temporal activation of a recovered source is given by $\hat{\mathbf{s i}}(\mathrm{t})$. This time series can be displayed as a plot of signal strength as a function of time or alternatively in an MEG image (e.g., Fig. 2h), a pseudo-colored bit map in which the responses of a given component during an entire experiment can be parsimoniously displayed. Typically, each row repre sents one discrete trial of stimulation and multiple trials are ordered vertically from top to bottom. MEG images will be used throughout this paper to provide temporal information, for displaying results from single-trial response onset time estimation.

\section{Spatial Aspects SO BI Components}

Although SOBI does not assume any physical model of the neuronal source generators, spatial information concerning a separated component is given by its sensor projection or field map, which represents the measured sensor response to the activation of the component $\hat{\mathbf{s}}_{\mathrm{i}}(\mathrm{t})$. The field map (e.g., Fig. $6 \mathrm{~b}$ ) of the ith component $\hat{\mathbf{s}}_{\mathbf{i}}(\mathrm{t})$ is the ith column of the estimated attenuation matrix $\hat{\mathbf{A}}$, where $\hat{\mathbf{A}}=\mathbf{W}^{-1}$. In combination with structural MRI images, the field maps can be used as input to standard tools for localizing the separated components within the brain (Tang et al., 2002). In this paper, we examined neuromagnetic source activation that can be modeled by ECDs.

\section{Single-Trial Response O nset Time Detection}

Single-trial response onset time detection is performed only when there is an evoked response that clearly deviates

\footnotetext{
${ }^{3}$ SOBI ICA algorithm produces the same number of components as there are channels in their input. For more details on SOBI application to MEG, see Tang et al. (2002), Methods section and Appendix B.2.

${ }^{4}$ These sensor projections may be best modeled by either dipoles or distributed sources.
}

from the baseline in the averaged component data. For all identified neuronal sources, we estimated response onset times by the leading edge of the response, rather than the time of the peak response. This measure better captures the intuitive goal of detecting the time of the earliest detectable response.

The process of single-trial response onset detection is iterative: both the threshold and the detection windows are adjusted until no further reduction in false detections and misses can be achieved. An initial threshold was set between the peak amplitude and one-half of the peak amplitude in the event-triggered average plot (not shown). The beginning of the detection window initially was set at the time the event triggered averages first exceeded the range of baseline fluctuation. Typically, the baseline window was approximately $100 \mathrm{~ms}$ prior to stimulus onset. The detection window ended when the event-triggered averages first returned to the same level as when the detection window began. (These initial values are not critical because they can be adjusted in both directions as described below.)

Because single-trial responses can be very different from the event-triggered averages, the threshold and detection windows were adjusted through an iterative process to ensure that no responses were excluded. For the components showing biphasic responses, the majority of single-trial re sponse time analyses presented here are performed on the initial phase of the response when the amplitude of the initial response is sufficiently large. Some results on later components are also shown when the early phase responses have relatively low amplitude. Using the initial threshold and detection window, response onset times were determined and graphically superimposed on the MEG image (detected response time (DRT) curve) to allow visual verification of the detected onset times.

\section{Threshold}

If the threshold is set too high not only can many trials remain undetected, but also one will overestimate the onset times by missing the initial onset. Overestimation of onset times is easily seen as a right shift in the DRT curve from the leading edge of color change associated with the responses (Fig. 2a). Because MEG images are generated in such a way that all trials with responses detected are displayed on top and sorted in increasing DRT, and all trials with no re sponses detected are displayed at the bottom, missing re sponses are apparent under visual inspection as shown at the bottom of Fig. 2a. If the threshold is set too low, false detection can occur when the amplitude of baseline fluctuation is relatively large. In this trial in which detection occurs exactly at the beginning of the response detection window will not be marked, therefore resulting in missing onsets from trials of correct detection (smooth curve), as in Fig. 2b. In both cases, the threshold could be either lowered or raised accordingly in the next iteration.

\section{Detection Window}

Once the detection threshold is determined, one further examines the MEG image for false detections associated with 
TABLE 1

Sample Detection Parameters and Results

\begin{tabular}{|c|c|c|c|c|c|}
\hline \multirow[b]{2}{*}{ Cases } & \multicolumn{3}{|c|}{ Detection parameters } & \multicolumn{2}{|c|}{ Detection results } \\
\hline & Threshold & $\mathrm{Wb}$ & We & Rate & Quality \\
\hline High threshold & 150 & 70 & 120 & $177 / 350$ & Missing onsets \\
\hline Low threshold & -100 & 70 & 120 & $97 / 350$ & Missing onsets \\
\hline Early Wb & 10 & 25 & 120 & $189 / 350$ & Missing and false onsets \\
\hline Late $\mathrm{Wb}$ & 10 & 95 & 120 & $91 / 350$ & Missing onsets \\
\hline Early We & 10 & 70 & 85 & $90 / 350$ & Missing onsets \\
\hline Late We & 10 & 70 & 320 & $313 / 350$ & False onsets \\
\hline Optimal & 10 & 70 & 120 & $305 / 350$ & \\
\hline
\end{tabular}

incorrect settings of the beginning and ending of the detection window, $\mathrm{Wb}$ and $\mathrm{We}$. If $\mathrm{Wb}$ is too early, the response window will include a part of the baseline and false detections similar to those caused by a low threshold (Fig. 2c). If $\mathrm{Wb}$ is too late, there will be trials in which response detections are apparently missing (see bottom portion of the MEG image) (Fig. 2d). If We is too early, responses may be missing (see bottom portion of Fig. 2e). This case can be identified using a method similar to that for identifying missing trials due to too high a threshold. If We is too late, the later portion of a biphasic response will be falsely detected as the initial response. This form of false detection is easily seen near the tail end of the DRT curve (Fig. 2f) where initial responses were clearly missed, while the second phase of the responses were marked. In any of the above cases, one can adjust the detection window parameters for the next iteration.

For each neuronal source, this iterative process continues until no further reduction in the frequency of false detections or missing responses can be achieved. Statistics on the detected onset times (mean \pm SEM) are then computed and reported along with the resulting MEG images presented in the order of increasing onset time or in natural trial order (Figs. $2 \mathrm{~g}$ and $2 \mathrm{~h}$ ). The sorted MEG image is the most useful in evaluating the result of onset detection. Therefore, the unsorted images are omitted from the figures in most cases. See Table 1 for associated detection parameters and results.

To determine whether the detected responses are above the background ongoing activity, such as alpha oscillation (Williamson et al., 1996), we applied the same procedure on a control window of equal size prior to or after the detection window ( $\mathrm{Wb}$ to $\mathrm{We}$ ) using otherwise identical parameters. The resulting number of onsets detected within such control windows can be compared statistically with that obtained for the response detection window. If single-trial ERPs above the background activity can be detected, then we expect that the detection rate for the response window will be significantly greater than that for the control window.

\section{SO BI Component versus Sensor Signals}

We evaluated whether better performance in singletrial response onsets measurement can be achieved with the aid of SOBI than using sensor signals directly. For each SOBI component that corresponds to the activation of a specific brain region, we applied the same single-trial analysis procedure to signals from a sensor that contained the largest peak responses over the ROI. For each such componentsensor pair, we first measured detection rate from detection windows of the same size and false detection rate from the control windows due to background noise, using the same detection procedure. We then calculated the difference between the two detection rates as a measure of detection rate for responses above the background activity. We performed a paired $t$ test on this final detection rate to determine if single-trial response onsets can be better measured from SOBI components than from sensors directly.

\section{RESULTS}

\section{Effect of Filter Length on Detected O nset Times}

As filtering can affect response onset times, we first investigated the effect of a low-pass filter, as such a filter is often used to remove noise unrelated to the evoked responses. Figures 3 and 4 display the result of response onset time detection using different low-pass filter parameters for a SOBI component with auditory evoked responses. Filtering visibly reduced the amount of background noise, thus highlighting the evoked responses (Fig. 3). There was no apparent change in the detected onset times or in the number of detections as the low-pass filter was changed from no filter to 40,20 , and $10 \mathrm{~Hz}$ (Fig. 3) or as the roll-off parameter was changed from 0.5 to $5 \mathrm{~Hz}$ (Fig. 4). A quantitative comparison between the detected onset times using different low-pass filter parameters revealed very small changes in the number of onsets detected. When a more aggressive low-pass filter was used, the number of events detected was reduced from to 145 to 141 (4 from a total of 150 trials).

If an onset time is detectable only when no filter is used, it is possible that such a detected response is a result of false detection due to noisy ongoing background activity. Therefore, by using a more aggressive low-pass filter, one can reduce the chance of false detection. On the other hand, a more aggressive filter can change the detected onset times. Thus, a change in the number of onsets detected caused by different low-pass filters could be a result of better onset time estimation associated with a reduction in false detection or, worse, estimation due to temporal 
(a)

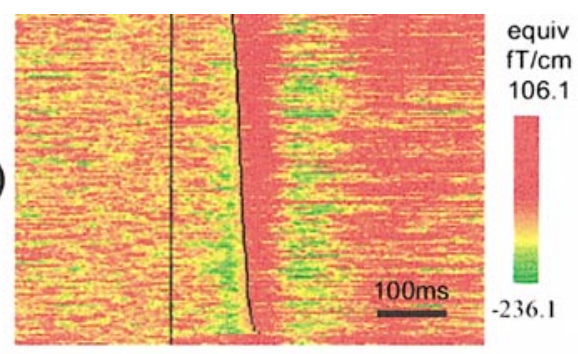

(c)

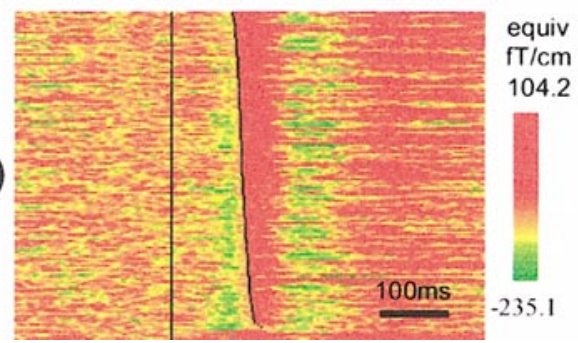

(b)

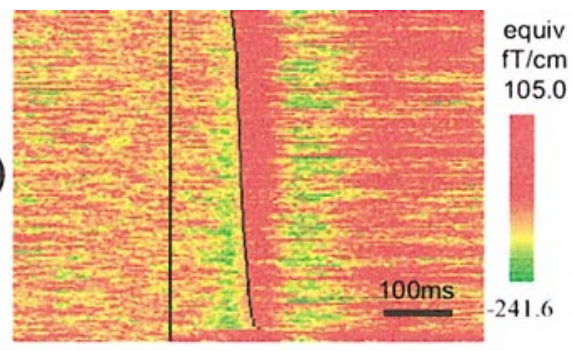

(d)

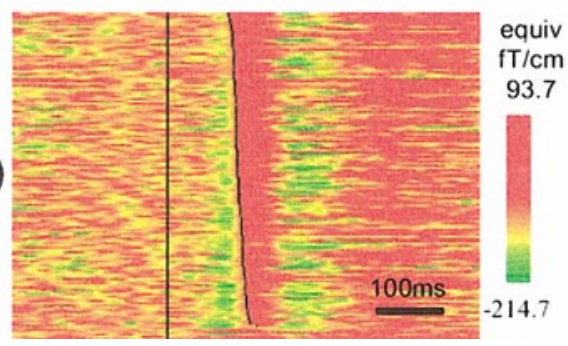

FIG. 3. Effect of low-pass filter on response onset time detection (onset times, \% response detected). (a) No filter, $90 \pm 1 \mathrm{~ms}$, $96.7 \%$; (b) low-pass at $40 \mathrm{~Hz}, 91 \pm 1 \mathrm{~ms}, 96 \%$; (c) low-pass at $20 \mathrm{~Hz}, 92 \pm 1 \mathrm{~ms}, 95.3 \%$; (d) low-pass at $10 \mathrm{~Hz}, 92 \pm 1 \mathrm{~ms}, 94 \%$. F or all images, a roll-off of $5 \mathrm{~Hz}$ was used.

smearing after filtering. To ensure that temporal smearing was not the cause of the change in the detected onset times, we always performed the detection procedure both with and without filtering and examined graphically whether the filtering altered the temporal profile of the evoked response. As shown in Fig. 3, filtering with a $10-\mathrm{Hz}$ low-pass filter changed the estimated onset times by less than $2 \mathrm{~ms}$ and did not distort the profile of the evoked responses.

(a)

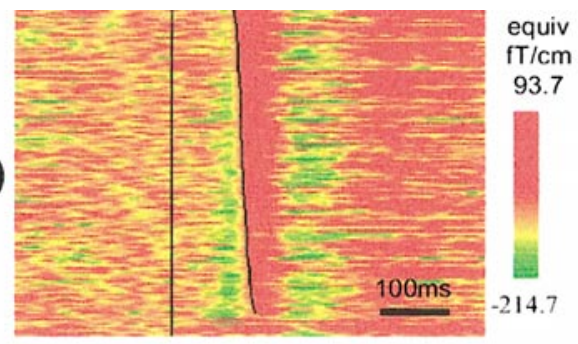

(c)

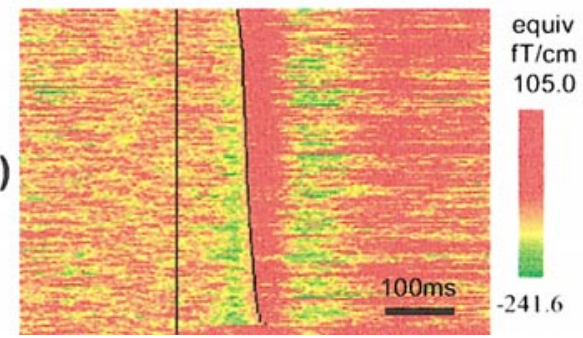

A more aggressive low-pass filter can reduce the influence of ongoing background activity, and thereby minimize false detection, without significantly altering the temporal profile of the evoked responses. Therefore, in the following analysis, a low-pass filter of $10 \mathrm{~Hz}$ with a roll-off of $5 \mathrm{~Hz}$ was used unless otherwise specified. It is important to note that for different neuronal sources, the effect of a given filter on response onset times will be different. When a filter significantly changes the temporal profile of the evoked responses, (b)

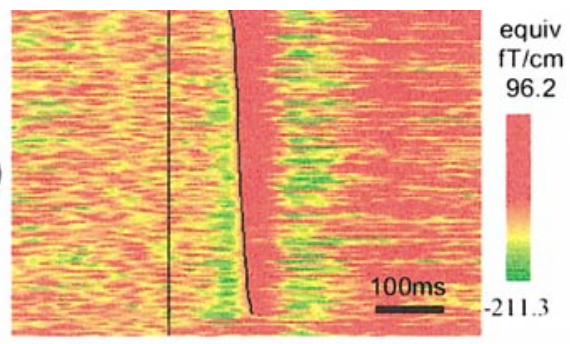

(d)

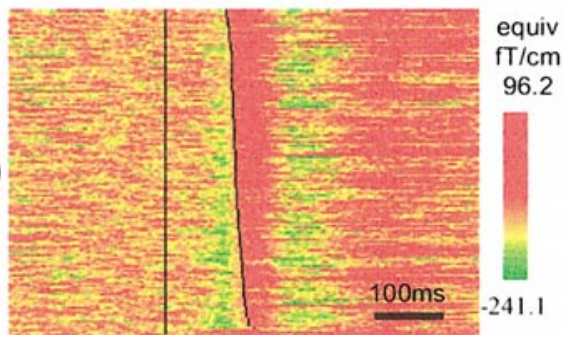

FIG. 4. Effect of filter roll-off on response onset time detection (onset times, \% response detected). (a) $10-\mathrm{Hz}$ low-pass and 5- $\mathrm{Hz}$ roll-off, $92 \pm 1 \mathrm{~ms}, 94 \%$; (b) 10-Hz low-pass and 0.5-Hz roll-off, $92 \pm 1 \mathrm{~ms}, 94 \%$; (c) 40-Hz low-pass and 5-Hz roll-off, $91 \pm 1 \mathrm{~ms}, 96 \%$; (d) 40-Hz low-pass and $0.5-\mathrm{Hz}$ roll-off, $91 \pm 1 \mathrm{~ms}, 96 \%$. 
(a)

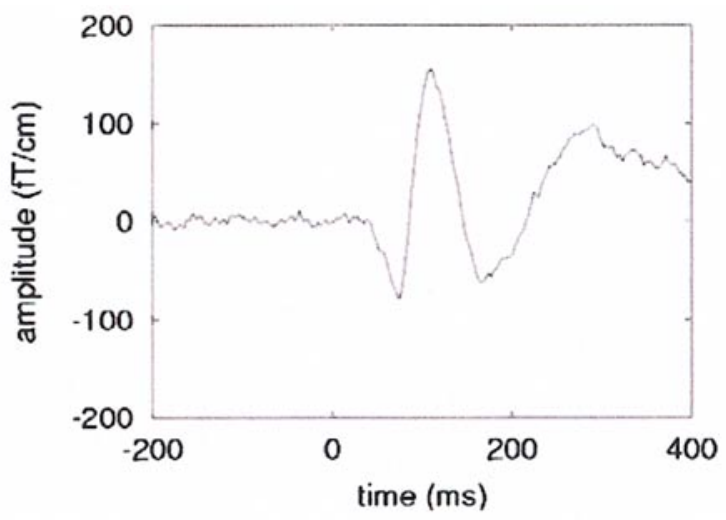

(b)

(c)
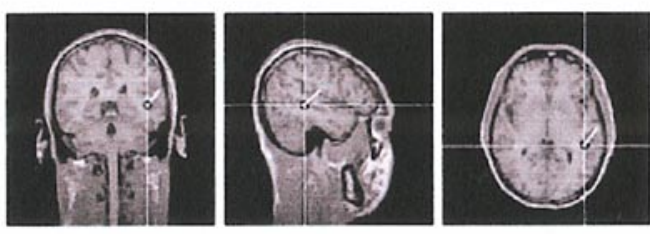

(d)
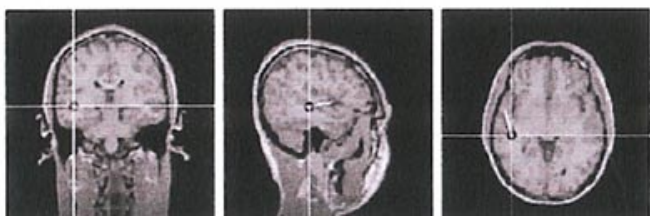

(e)

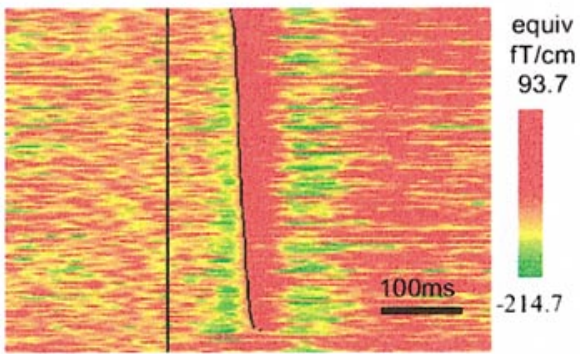

(f)

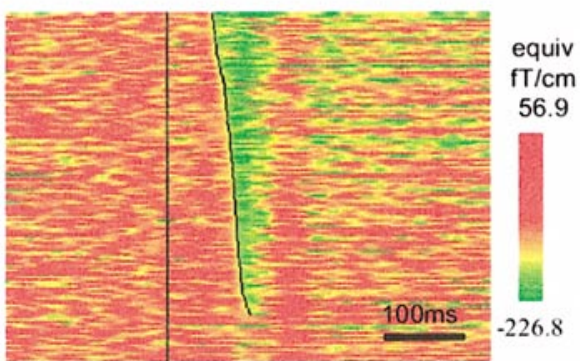

(g)

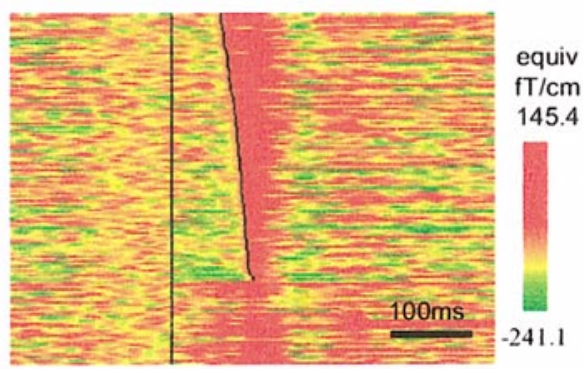

(h)

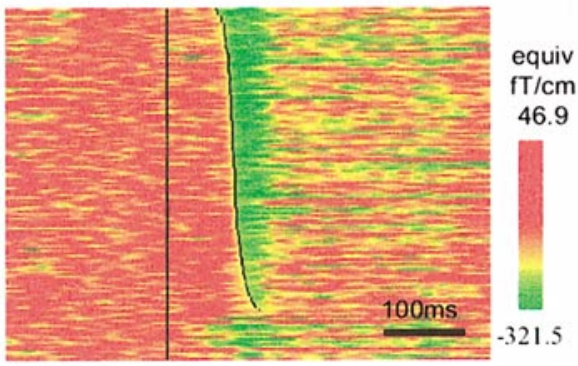

FIG. 5. Detection of singletrial response onset times from an auditory source. (a) Auditory stimulus-triggered average response (unfiltered). (b) Field map of the temporal source. (c and d) Fitted ECD superimposed on the subject's structural MR images. (e) Detected single-trial response times marked on an MEG image. (f- $h$ ) Single-trial auditory response onset detection across three additional subjects, sorted by onset latency. $(\mathrm{e}-\mathrm{h})$ Subjects $1-4 . \mathrm{N}=150$ trials.

a less aggressive filter should be used for an accurate estimation of response onsets.

\section{Response 0 nset Time Detection across Sensory Modalities}

In this section we demonstrate that single-trial response onset time detection can be achieved in three major sensory modalities and under experimental conditions of both large and small trial-to-trial variability. Single-trial onset time detection with small trial-to-trial variability was performed for the auditory-evoked responses recorded during simple sensory activation (binaural pure tone presentation). Single- trial onset time detection with large trial-to-trial variability was performed for the visual and somatosensory evoked responses recorded during the cognitive tasks.

The detected response onset times are shown in MEG images (Fig. 6d), with the evoked responses aligned to the stimulus onset (time zero, marked by the vertical line on the left side of the MEG image) and the detected response times marked as a curve to the right of the stimulus onset line (DRT curve). The detection results are shown sorted by latency from stimulus to detected response onset. The stimulus-triggered average (Fig. 6a), sensor projections or field 
maps (Fig. 6b), and dipole location superimposed on the subject's structural MR images (e.g., Fig. 6c) are also provided, for comparison with results from standard analysis. For the visual source shown in Figs. 6a- $6 \mathrm{~d}$, the single-trial response onsets were detected in 64 of 90 trials (71.1\%). The estimated onset times were $111 \pm 1 \mathrm{~ms}$. Its temporal profile in the average response, the field map, the contour plot, and the dipole location were characteristic of typical visual sources from the occipitoparietal lobes. For comparison with typical visually evoked responses, see Brenner et al. (1975), Hari (1994), and Supek et al. (1999).

For the auditory source shown in Figs. $5 a-5 e$, the singletrial response onsets were detected in 141 of 150 trials (94\%). The estimated response onset time was $92 \pm 1 \mathrm{~ms}$. The temporal profile in the average response, field map, contour plot, and dipole location were characteristic of typical auditory sources. This particular auditory SOBI component had a two-dipole solution, one in each of the two hemispheres and (necessarily, due to the SOBI decomposition) both having the same time course of response. This is consistent with the binaural stimulation used in this experiment. ${ }^{5}$ The temporal profile in the average response, the field map, the contour plot, and the dipole location were characteristic of typical auditory sources in the temporal lobes. For comparison with typical auditory-evoked responses, see Hari \& al. (1980), Romani et al. (1982), and Roberts et al. (2000).

For the somatosensory source shown in Figs. 7a-7f, the single-trial response onsets were detected in 129 of 150 trials $(86 \%)$ when the contralateral thumb pressed the mouse button and in 105 of 120 trials (87.5\%) when the ipsilateral thumb pressed the mouse button. The response onset times from the time when the button press was detected on the trigger line were $-1 \pm 2$ and $15 \pm 1 \mathrm{~ms}$ for the contra- and ipsilateral activation, respectively. These numbers indicate that the somatosensory responses could start as soon as the thumb movement was initiated-as soon as, or even before, the mouse button was completely depressed. The temporal profiles in the average responses were slower to rise and broader in width than the typical responses evoked by electrical stimulation (Brenner \& al., 1978; Hari and Forss, 1999; Karhu and Tesche, 1999). This was expected because somatosensory stimulation due to button-press movement and feedback is much more prolonged and variable than stimulation by the brief and well-controlled median nerve shock. The field map, contour plot, and dipole location were consistent with activation of the hand region of the somatosensory cortex.

\section{Cross-Subject Response O nset Detection: Auditory}

Auditory-evoked responses from the presentation of a pure tone were the least variable in comparison to the above described visual and somatosensory responses from the cognitive tasks. In all six subjects, auditory sources can be identified and localized from the SOBI separated compo-

\footnotetext{
${ }^{5}$ It is possible to obtain two separate components from the left and right hemispheres if there is sufficient hemispherical asymmetry in the temporal details of neuronal responses from the left and right auditory cortices.
}

nents. Single-trial response onset time detection could be performed in six of six expected auditory sources ${ }^{6}$ with a detection rate of $80 \pm 5 \%$ and estimated response onset times of $85 \pm 1 \mathrm{~ms}(\mathrm{~N}=6)$.

Figures $5 \mathrm{f}-5 \mathrm{~h}$ show results of onset detection for the auditory source from three additional subjects. As the trial-totrial variability in auditory stimulation was very low in comparison to the variability in the visual and somatosensory stimulation during the cognitive tasks, the average detection rate was higher for these auditory sources. Furthermore, single-trial response onset time detection could be performed among a higher percentage of expected sources (100\%) for the auditory responses than for the visual (81\%) and somatosensory $(29 \%)$ responses.

\section{Cross-Subject Response O nset Detection: Visual}

As previously discussed (Tang et al., 2000b, 2002), visual sources were identifiable along both the ventral and the dorsal streams. The occi pitoparietal sources along the dorsal stream varied less in location and in response profile. In contrast, the occipitotemporal sources along the ventral stream showed greater variability in response profile and precise location. To give the readers a sense of how well the single-trial onset time detection procedure can perform across a variety of visual sources, we show detection for the visual responses from a variety of visual areas from multiple subjects.

In 13 of 16 (81\%) expected visual sources along the ventral processing stream, ${ }^{7}$ single-trial onset time detection could be performed. The detection rate was $69 \pm 2 \%$, and the estimated response onset time was $139 \pm 9 \mathrm{~ms}(\mathrm{~N}=13)$. Figures $6 e-6 g$ show results of onset time detection for the visual sources from three additional subjects. Sources were chosen to reflect variability in the responses and in the detection.

\section{Cross-Subject Response 0 nset Detection: Somatosensory}

Somatosensory sources were identified in all subjects who made button-press responses during the four cognitive tasks. Single-trial response onset time detection was attempted on one of the SOBI somatosensory components for each subject in at least one of the four tasks. Because the activation of these somatosensory sources was highly variable, in only 7 of $24(29 \%)$ of the somatosensory sources could single-trial onset time detection be performed. Among these sources, for the contralateral button presses, single-trial onset times were estimated to be $0 \pm 3 \mathrm{~ms}$ with a detection rate of $81 \pm 2 \%$ $(\mathrm{N}=7)$. For the ipsilateral button presses, single-trial onset times were estimated to be $5 \pm 3$ ms with a detection rate of $76 \pm 5 \%(\mathrm{~N}=3)$. Figures $7 \mathrm{~g}$ and $7 \mathrm{~h}$ show results of onset time detection for the latter source in two additional subjects.

\footnotetext{
${ }^{6}$ Given that the tasks involved auditory stimulation, we expected at least one auditory source to be activated in each subject. A total of six auditory sources were therefore expected for the six subjects.

${ }^{7}$ Given that the tasks involved memory of visual forms, we expected at least one visual source to be activated along the ventral processing pathway. A total of 16 such sources were expected for four experiments on four subjects.
} 


\section{Single-Trial Response 0 nsets and Background Activity}

To determine quantitatively whether the detected re sponses are due to baseline ongoing activity, we performed the detection procedure on the detection window $(\mathrm{Wb}$ and We) and on a baseline or control window of equal length immediately before and after the response window, using otherwise identical parameters. The number of detections obtained from the control window was considered to reflect background ongoing activity (also referred to as false detections). When a postresponse control window was selected, we made sure that the background fluctuation was comparable to or greater than that of the prestimulus baseline. Figure 8 shows the result of response onset time detection for the response window, a preresponse control window, and a postresponse control window. The trials are sorted according to the detected onset times in Figs. $8 \mathrm{a}, 8 \mathrm{c}$, and $8 \mathrm{e}$ and the same detection results are shown in natural trial order in Figs. $8 \mathrm{~b}$, $8 d$, and $8 f$.

The detection rates for the response windows were $78 \pm 2 \%$ $(\mathrm{N}=8)$ for the somatosensory components, $69 \pm 2 \%(\mathrm{~N}=13)$ for the visual components, and $80 \pm 5 \%(N=6)$ for the auditory components. When all sources were pool ed across all modalities, the detection rate within the detection window was $74 \pm 2 \%$. The detection rates obtained for the control windows using otherwise identical parameters were $27 \pm 4 \%$ for the somatosensory components, $27 \pm 3 \%$ for the visual components, and $23 \pm 3 \%$ for the auditory components. When all sources were pooled, the false detection rate across all modalities within the control window was $26 \pm 2 \%$.

To determine whether the detected response onsets were more numerous than those detected in the control windows, we performed a t test on the difference between the number of detections during the response window and the number of detections during the control windows. We found that a significantly greater number of response onsets were detected from the response window than from the control window (somatosensory $(\mathrm{t}(7)=8.872, \mathrm{P}<0.0005)$, visual $(\mathrm{t}(12)=$ 11.054, $\mathrm{P}<0.005)$, auditory $(t(5)=9.288, \mathrm{P}<0.0005)$, overall $(\mathrm{t}(26)=16.145, \mathrm{P}<0.0005))$. The numbers of detections that were above the background activity were $52 \pm 6 \%$ for somatosensory components, $42 \pm 4 \%$ for the visual components, and $57 \pm 6 \%$ for the auditory components. When all sources were pooled across modalities, the rate of detection that was above the false detection rate was $48 \pm 3 \%$. These results indicate that our method is capable of detecting evoked responses from single-trial MEG data that are above background ongoing activity.

\section{SO BI Advantage in Single-T rial O nset Measurement}

To evaluate SOBI performance, we compared response onset time detection from SOBI components and detection directly from their corresponding sensors. Comparison of detection rates from the response windows ( $F$ ig. 9a) showed a significantly higher overall detection rate for the SOBI components than for the sensors (component $74 \pm 2 \%$, sensor $65 \pm 2 \%, \mathrm{t}(26)=4.598, \mathrm{P}<0.0005)$. For somatosensory stimulation with the highest variability, analysis of SOBI components yiel ded significantly greater detection rates than that obtained from the sensors (component $78 \pm 2 \%$, sensor $66 \pm 3 \%, \mathrm{t}(7)=4.967, \mathrm{P}<0.005) .{ }^{8} \mathrm{~F}$ or the visual stimulation with the second highest variability, analysis of SOBI components also yielded significantly greater detection rates than that obtained from the sensors (component $69 \pm 2 \%$, sensor $60 \pm 3 \%, \mathrm{t}(12)=3.223, \mathrm{P}<0.005)$. For the auditory stimulation with the least variability, analysis of SOBI components did not yield significantly greater detection rates than that obtained from the sensors (component $80 \pm 5 \%$, sensor $77 \pm 5 \%, t(5)=0.653, P>0.2)$.

Comparison of false detection rates from the control windows (Fig. 9b) showed a significantly lower overall rate for the SOBI components than for the sensors (component $26 \pm$ $2 \%$, sensor $35 \pm 2 \%, \mathrm{t}(26)=3.671, \mathrm{P}<0.0005)$. For somatosensory stimulation with the highest variability, analysis of $\mathrm{SOBI}$ components yielded significantly lower false detection rates than that obtained from the sensors (component $27 \pm$ $4 \%$, sensor $40 \pm 2 \%, t(7)=2.638, P<0.025)$. F or the visual stimulation with the second highest variability, analysis of SOBI components al so yiel ded significantly lower false detection rates than that obtained from the sensors (component $27 \pm 3 \%$, sensor $36 \pm 4 \%, \mathrm{t}(12)=2.406, \mathrm{P}<0.05)$. For the auditory stimulation with the least variability, analysis of SOBI components did not yield a significant difference in false detection rates compared to that obtained from the sensors (component $23 \pm 3 \%$, sensor $27 \pm 3 \%, t(5)=1.166$, $P>0.2$ ). False detection rates from the sensors were higher for the modality of stimulation with the highest stimulation variability, ranging from 27 to $40 \%$ across the three modalities. In contrast, false detection rates remained relatively constant for the SOBI components, ranging from 23 to $27 \%$.

Detection rates that are above the false detection ( $\mathrm{No}$. of detections in the response window - No. of detections in the control window) (Fig. 9c) were significantly greater for the SOBI components than for the sensors $(\mathrm{t}(26)=5.045, \mathrm{P}<$ 0.0005 , Fig. 9). For somatosensory stimulation with the highest variability, analysis of SOBI components yielded significantly higher final detection rates ( $26 \pm 6 \%$ higher) than that obtained from the sensors $(t(7)=4.036, P<0.005)$. For the visual stimulation with the second highest variability, analysis of SOBI somatosensory components also yielded significantly greater final detection rates (18 $\pm 5 \%$ higher) than that obtained from the sensors ( $\mathrm{t}(12)=3.711, \mathrm{P}<0.005)$. F or the auditory stimulation with the least variability, analysis of SOBI components did not yield a significant difference in final detection rates $(7 \pm 8 \%)$ in comparison to that obtained from the sensors $(t(5)=0.927, P>0.2)$. The difference in detection rates between SOBI components and sensors that are above background activity was the highest for the somatosensory stimulation with the highest variability and the lowest for the auditory stimulation with the least variability.

\footnotetext{
${ }^{8}$ To increase statistical power for this component vs sensor comparison, we combined contra- and ipsilateral stimulation in the onset time detection.
} 
(a)

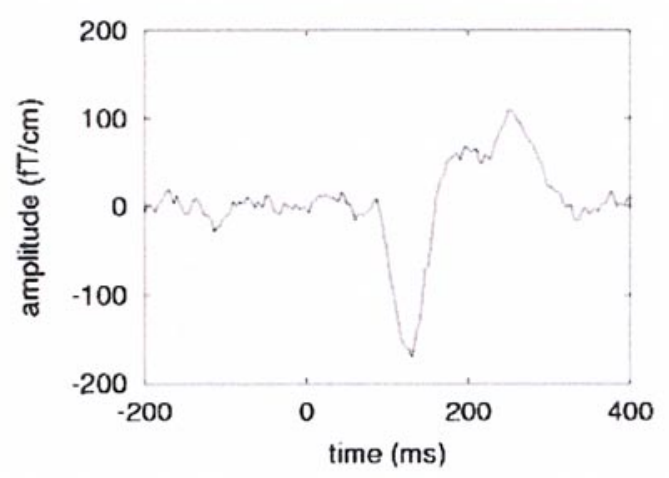

(b)

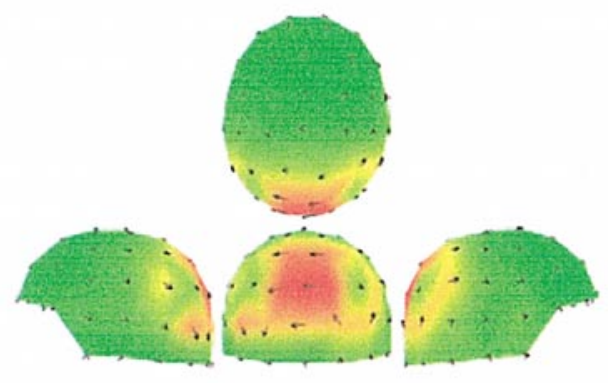

(c)

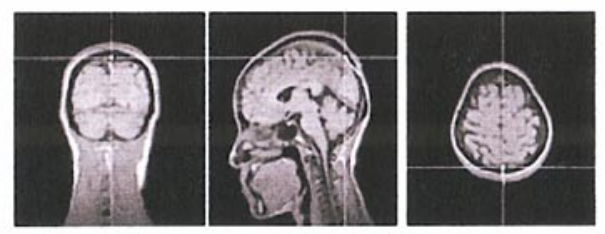

(d)

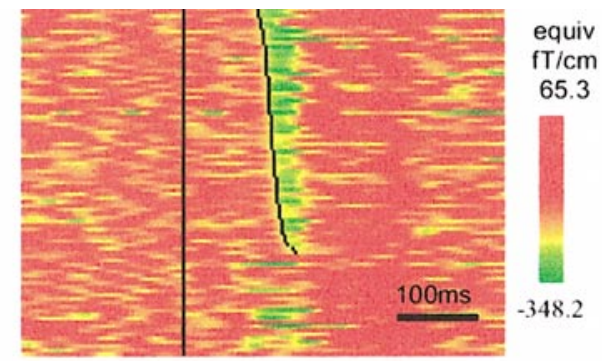

(e)

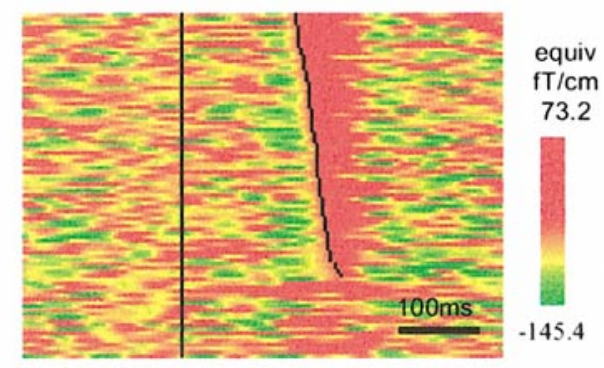

(f)

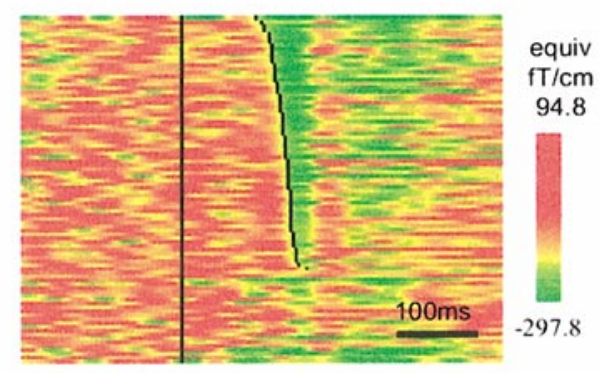

(g)

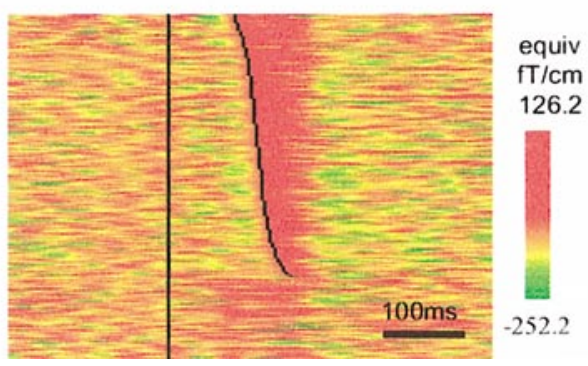

FIG. 6. Detection of singletrial response onset times from a occipitoparietal source that responded to a visual stimulus. (a) Visualstimulus-locked average response (unfiltered). (b) Field map of the parietal source activation. (c) Fitted ECD superimposed on the subject's structural MR images. (d) Detected single-trial response times marked on an MEG image. (e-g) Single-trial visual response onset detection in visual sources across three additional subjects, sorted by onset latency. (d-g) Subjects 1- $4 . \mathrm{N}=90$ trials except for (d) $\mathrm{N}=270$ trials.

\section{DISCUSSION}

\section{Summary}

Previously, we have shown that SOBI ICA can decompose the mixture of neuromagnetic and noise signals into multiple components. Each component has a continuous time course and a fixed sensor projection. The time courses and sensor projections of task-related components correspond to neuromagnetic source activations of specific brain regions as would be predicted by the specific tasks. Because SOBI can separate different noise and neuromagnetic signals from each other, by measuring single-trial ERPs from the time course of a
SOBI component, as opposed to from sensors directly, it was predicted that one should be able to improve single-trial response measurement from the corresponding neuronal source activation without the interference from overlapping noise signals and signals from other brain regions.

In this study, we applied SOBI to MEG data collected during stimulation of visual, auditory, and somatosensory modalities, each with varying degrees of variability in the stimulus presentation. We compared response onset detection results from SOBI components and their corresponding sensors. Applying the same threshold-crossing procedure to component and sensor data, we found a significantly higher 
(a)

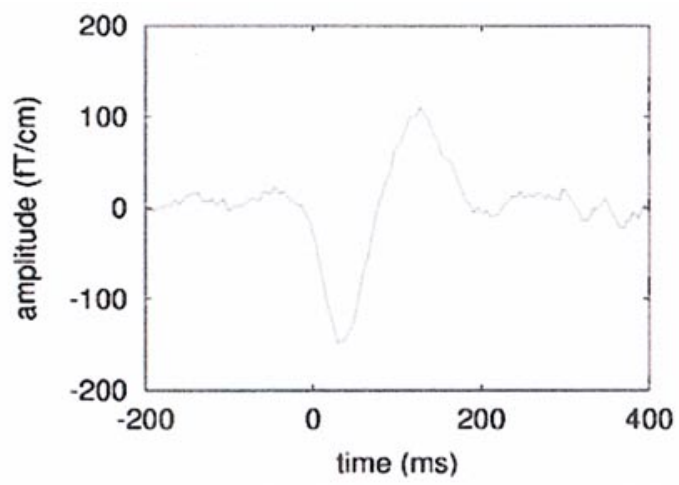

(b)

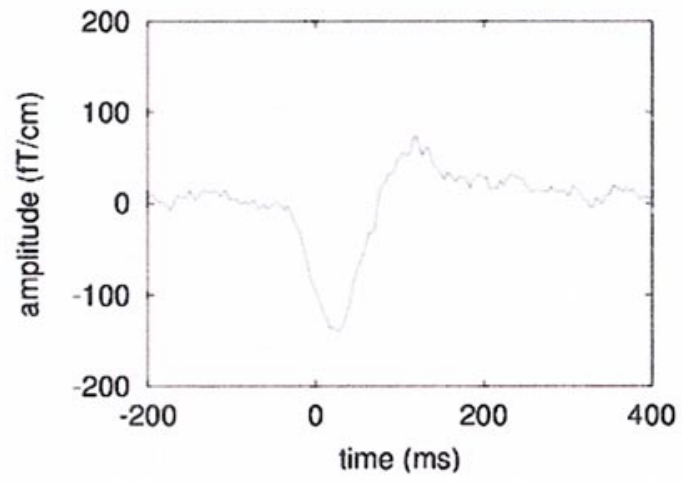

(c)

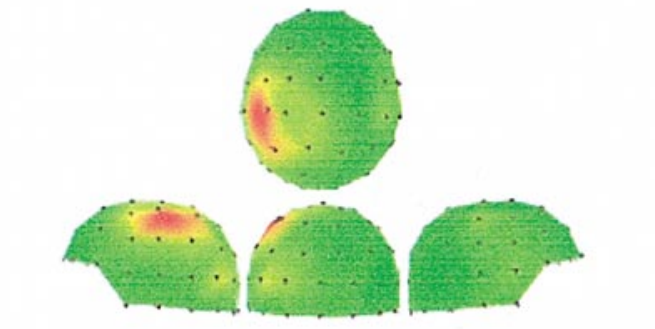

(d)

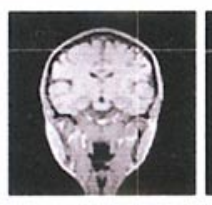

(e)

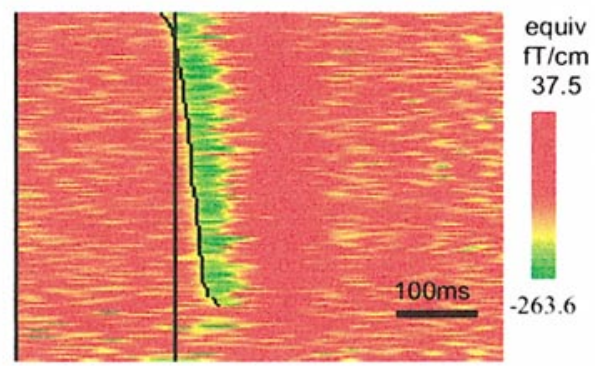

(f)

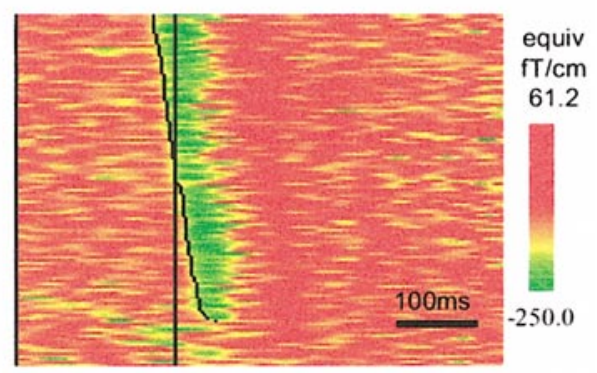

(g)

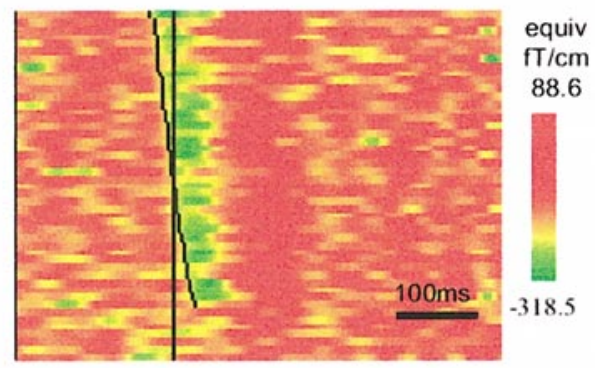

(h)

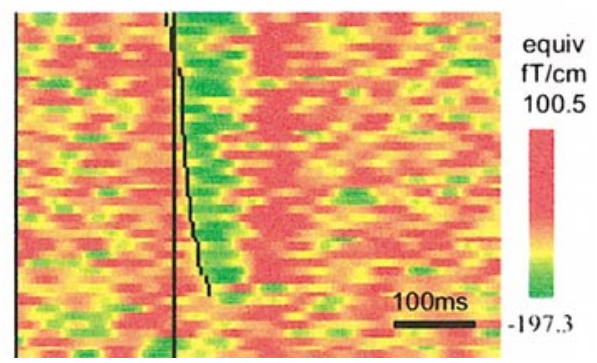

FIG. 7. Detection of single-trial response onset times from a somatosensory source that responded to left (a) and right (b) button presses. ( $a$ and b) Left and right button-press-triggered average responses (unfiltered). (c) Field map of the somatosensory source. (d) Fitted ECD superimposed on the subject's structural MR images. (e and f) Detected single-trial response onset times triggered by left and right button presses, respectively, marked on MEG images. ( $g$ and $\mathrm{h}$ ) Single-trial somatosensory response onset detection across two additional subjects, sorted by onset latency. (e-h) Subjects 1-3. Shown for contralateral activation only. The number of trials varied from subject to subject.

overall detection rate and a significantly lower overall false detection rate for the SOBI components than for the sensors. This SOBI advantage was most apparent when the variability in stimulus presentation was high and absent when the variability in stimulus presentation was small.

These results suggest that SOBI offers advantages not only in magnetic source localization under conditions of large trialto-trial variability (Tang et al., 2002) but also in response onset measurement from single-trial ERP. This combined improve- ment in source localization and singletrial response measure ment may open up opportunities for addressing new scientific questions that rely on the capability in measuring single-trial response onsets from specific brain regions.

\section{Single-Trial Analysis of MEG Data: Temporal Aspects}

In most MEG studies, response latencies, typically peak response latencies, were measured only from averages of 
hundreds of trials. Even when known MEG artifacts (eye blinks, heartbeats, metal in the teeth, etc.) are absent and when the total number of neuromagnetic sources are minimized, as in in vitro MEG experiments performed on hippocampal slices, averages of 300-600 trials were once needed (Kyuhou and Okada, 1993). Later, with a number of technical improvements, large amplitude epileptiform activity could be measured in continuous data (Okada and $\mathrm{Xu}$, 1996). Therefore, it should be clear that visualizing and measuring single-trial evoked responses in vivo from human subjects in the presence of many noise sources is a nontrivial task.

Despite the difficulties, MEG researchers have attempted to obtain temporal measurements from single-trial data. In contrast to our effort in applying SOBI to the detection of sensory evoked response onsets, Wübbeler et al. (2000), Ziehe et al. (2000), and Mackert et al. (2001) have applied SOBI to the analysis of DC signals from MEG data. From MEG data collected during alternating periods of prolonged resting and finger movement ( $30 \mathrm{~s}$ each), they were able to extract an ICA component whose sensor projection clearly corresponds to that associated with activation of motor cortex. Most impressively, the single-trial plot of the time course for this component showed unambiguous movement-related changes. Because this movement-related component was active during a prolonged period of time, the underlying source distribution, as one would expect, is too complex to be model ed by an ECD. Application of source-modeling algorithms that can handle distributed sources, such as magnetic field tomography (I oannides et al., 1995), to this component may be particularly helpful in offering spatial characterization of the underlying source distribution. Nevertheless, this result, together with our findings, provides support that the SOBI ICA algorithm allows temporal characterization of single-trial MEG data.

Stufflebeam et al. (2000) measured the physiological jitter in the single-trial latency of M100 of the auditory-evoked fields using a method based on correlation coefficients. The correlation coefficient computed from single-trial data was used as a measure for the temporal similarity among the latencies of M 100 across many single trials. Using this measure, they were able to show that adding noise to auditory input can lead to enhanced evoked auditory response, thereby demonstrating a cortical manifestation of the stochastic resonance phenomenon. In addition, they were able to extract features in the single-trial neuromagnetic responses that were not apparent in the averaged evoked response. Although an ICA algorithm was not used in their study, we would predict that preprocessing of the same data using an ICA algorithm would improve the $\mathrm{S} / \mathrm{N}$ ratio and thus offer a less contaminated measurement of the auditory-evoked re sponse reliability.

\section{Single-T rial Analysis of MEG Data: Spatial Aspects}

The first sustained efforts to perform MEG data analysis using single-trial data as input were carried out by l oannides et al. (1995, 2000b), Liu and Ioannides (1996), Liu et al. (1999), and Ioannides (2001) using magnetic field tomogra- phy (MFT). This method offers the advantage of modeling the spatial distribution of underlying neuronal sources, which may not be best modeled by the more commonly used ECDs, and of simultaneously deriving the corresponding time course for the underlying source distribution. It has been applied to both sensory (Liu and I oannides, 1996; I oannides, 1998) and cognitive tasks (Liu et al., 1999; Ioannides et al., $2000 a, b)$. Using this method, the authors were able to show distributed source activation at a particular point in time (single-trial analysis) (Ioannides, 2001).

In principle, the response onset times similar to those measured in our present study could be extracted from the time course of the MFT-estimated magnetically nonsilent current density at a particular spatial location. To our knowledge, estimating single-trial response latencies has not been a focus of any MFT study to date. As pointed out by l oannides (2001), what makes MEG single-trial analysis possible is a combination of improved recording technology and better signal processing methods for noise reduction. Ther efore, the use of ICA methods, such as SOBI, can be viewed as a more advanced noise reduction method that not only separates various known noise sources from the brain-related signals but al so separates brain signals from each other. In this view, SOBI, and ICA in general, could be used to provide multiple functionally independent inputs with improved $\mathrm{S} / \mathrm{N}$ ratios as inputs to the MFT method.

A major difference between SOBI, the ICA method used in the present study, and MFT, is that SOBI separates a neuronal source from noise sources and from other neuronal sources using detailed temporal information while MFT uses spatial information at one point in time to estimate the current density at that moment. This selective use of different types of information (temporal vs spatial) suggests that the two methods may be mutually beneficial if used in combination. For example, SOBI can be used to generate cleaned-up components that correspond to functionally independent units, to which MFT can be subsequently applied to estimate the spatial distribution of these SOBI components. SOBI can also take advantage of other source localization methods by serving as a front end. Single- or multiple-dipole methods and point or distributed source modeling methods (Huang et al., 1998a,b; I oannides et al., 1995) can all be used on ICA's output. Potential benefits of combining SOBI with MFT or other non-dipole-based distributed source modeling methods remain to be explored.

Because SOBI ICA does not use Maxwell's equations and does not attempt to physically model the sources, SOBI does not suffer from two types of potential errors: (1) numeric errors in the forward model and (2) errors arising from assumptions made in the spatial domain, such as how distributed a neuromagnetic source is. ${ }^{9}$ Because the forward process is quite ill conditioned, even small errors in a forward model lead to large errors in al gorithms that first estimate a forward model and then invert it. This means that a forward

\footnotetext{
${ }^{9}$ Instead, ICA's estimation errors are most likely associated with assumptions made in the temporal domain. See the discussion of assumptions.
} 
model might be of greater utility for checking whether the results of a blind method are consistent with the physics than for constraining the results of a recovery algorithm. Moreover, when using ICA, identification of functionally interesting neuronal sources is not limited by whether the activated neuronal populations can be modeled by point or distributed sources. One can measure response onset times and other temporal characteristics of responses from discontiguous neuronal populations, as long as they show coherent activation.

\section{Response O nset Time Detection}

Typical parameters estimated for a single-trial evoked response include peak amplitude and peak latency. Although the earlier template matching methods allow an arbitrary response profile to be used as a template, they required the response template to fit every single-trial response (Woody, 1967), even when an adaptive process was used to allow updating of the template from iteration to iteration (Chung et al., 1996). One methodological improvement over earlier template matching (Woody, 1967) and maximum likelihood methods (Pham et al., 1987) was to simultaneously estimate single-trial response amplitude and latency (J askowski and Verleger, 1999). Even with this improvement, the duration of the evoked response was held constant. As one can see from visual inspection of the MEG image of singletrial data, the duration of an evoked response changes widely, with some sustained for as long as $70 \mathrm{~ms}$ and others as short as $20 \mathrm{~ms}$ (e.g., Fig. 7f). Therefore methods assuming fixed duration may not work well when the response duration is not constant. In this paper, instead of developing more complex models for single-trial response estimation, we used a simple method for the purpose of evaluating the advantage SOBI can offer for detecting single-trial response onset time. This method does not require any assumptions about the rest of the response parameters. In doing so, we avoided the issue of variability in amplitude, peak latency, duration, and the precise waveforms of the evoked responses.

It is important to note that because real data were used, this study cannot offer performance measures comparable to those of previous methodological studies using synthetic data. Instead, the detected single-trial response onset times are evaluated by both visual inspection and statistical comparison between event detection in a response window vs a control window. First, the iterative process described in this paper allows adjustment of threshold and detection windows to minimize apparent misses and false detection. Second, statistical tests can be performed to address the issue of whether the number of detected response onsets during a response window is significantly greater than the number of detections when the same process is applied to a control window of equal size. Multiple control windows can be se lected from both before and after the response window. For the ICA components described in this paper, the number of single-trial responses detected during the response window was significantly greater than that during the control window.
To fully benefit from the capability of measuring singletrial response onset, it is critical that the response onset be detected in a reasonable proportion of the trials. Therefore, it is important to point out that the proportion of the trials in which the response onset times can be estimated depends on a number of factors. Low detection rates can result from poor separation, which gives rise to residual sensor and environmental noise in the separated neuronal components. It can also result from poor execution of the detection procedure. Most importantly, a low detection rate can be a direct consequence of non-ICA factors, including a high amplitude of baseline neuronal signal fluctuation, a high amplitude of baseline neuronal oscillatory activity, a high variability in the stimulus presentation, a high variability in the neuronal source activation due to the specific behavioral task, or other sources of noise that decrease the $\mathrm{S} / \mathrm{N}$ ratio.

\section{Knowing, Dealing with, and Using the Bias}

The current single-trial response onset detection method has a clear bias, that is, it includes onsets of events that are not necessarily above the background activity. In the previous paragraph, we discussed how one can easily determine statistically whether and how many of the detected events are above the background activity. Here, we present some advantages such a bias can offer and some risks associated with eliminating this bias. In developing the current procedure for onset time detection, we considered the option of eliminating some of the detected response onsets if the amplitude at the time of detection was close enough to the baseline fluctuation. This option appears to have the advantage of minimizing the number of false detections. Yet, it would lead to less informative or misleading measurement for addressing subsequent scientific questions.

We offer two cases to illustrate the point. First consider two neuronal populations $A$ and $B$, both of which send inputs to neuronal population C. Assuming the most extreme case in which every single-trial evoked response onset detected in A and $B$ within a common response window is not above and beyond the baseline rhythmic activity. Yet, being able to measure the "onset" of one cycle of such rhythmic activity in neuronal sources $A$ and $B$ in the shared time window allows one to address scientific questions concerning how the relative timing of multiple input signals from $A$ and $B$ can affect subsequent neural information processing taking place in $\mathrm{C}$. The fact that these types of questions can be answered by using different methods does not constitute a good reason for throwing out such useful information easily offered by our current detection procedure.

Second, in estimating single-trial response latency, one needs to determine not only when a response occurred but whether a response occurred, as well (response reliability). As is always the case, in some trials with high $\mathrm{S} / \mathrm{N}$ ratio, there is no question when a response occurred and good estimation can be achieved. However, when the $\mathrm{S} / \mathrm{N}$ ratio is poor, the performance of an algorithm or method can deteriorate. One could eliminate those low $\mathrm{S} / \mathrm{N}$ trials (null trials) from the analysis to achieve better performance, as was often the practice in the EEG literature (Smulders \& al., 1994; 
a

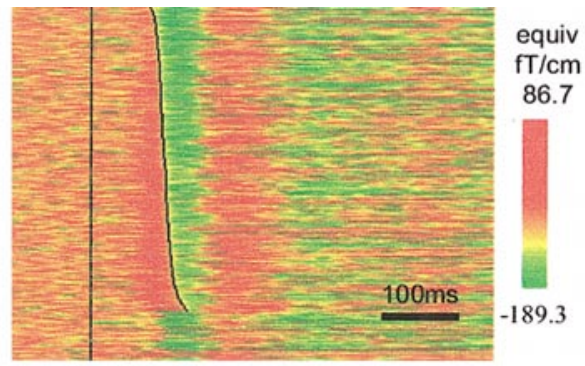

C

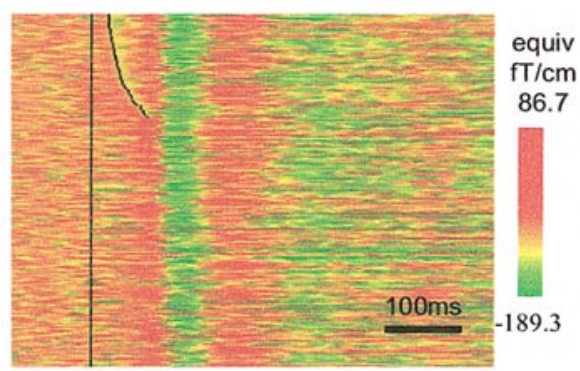

e

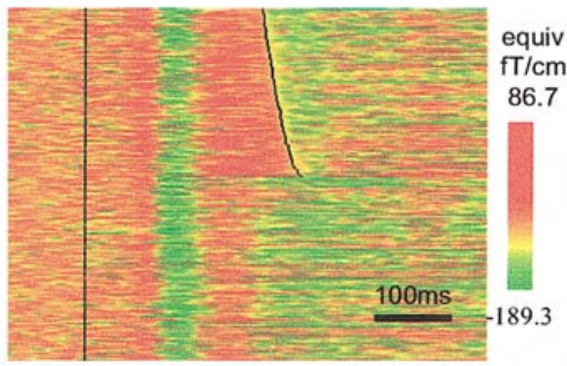

b

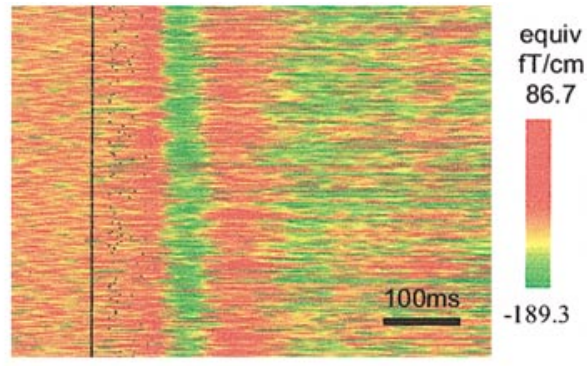

d

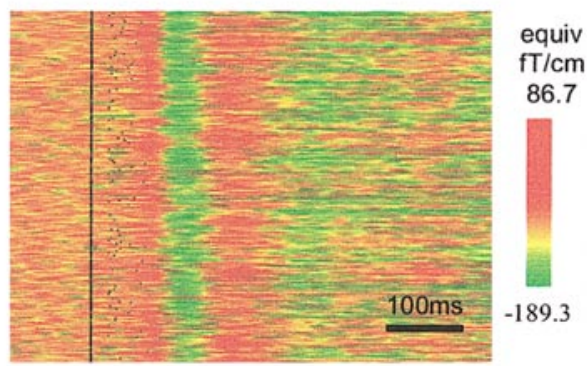

f

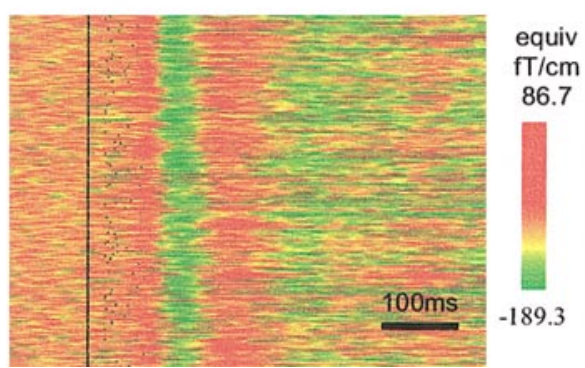

FIG. 8. Detected response onsets for the response and pre- and postresponse control windows in (a and b) response window; (c and d) precontrol window, (e and f) postcontrol window. (a, c, e) Trials sorted by increasing onset latency. (b, d, f) Trials in natural order.

Ford et al., 1994). However, there is a price to pay for such an improvement: a bias against single-trial responses of smaller amplitude (J askowski and Verleger, 2000) and a general bias with respect to variables that are of interest to the experimenter (Smulders et al., 1994). It is possible that the smaller response amplitude and reduced response reliability are associated with a subject's specific responses to a particular experimental manipulation. By eliminating such small responses (not so far above the background fluctuation) from the analysis, one can potentially draw incorrect conclusions. Therefore, by keeping the seemingly false detection as part of the measurement but further comparing the same measurement with that obtained for a control window (Ford et al., 1994; J in et al., 1997) allows one to study how an experimental manipulation can influence single-trial evoked responses without throwing out potentially relevant information.

\section{Filtering}

As thoroughly reviewed by J askowski and Verleger (2000), at least for $\mathrm{P} 3$ response estimation, empirical results showed that single-trial response latency estimation using a filter setting of $3.5 \mathrm{~Hz}$ was optimal for the peak picking method, cross-correlation- and covariance-based methods, and various maximum likelihood methods (Pfefferbaum et al., 1984, 1986; Fein and Turetsky, 1989; Michalewski et al., 1986; Smulders et al., 1994). I n using a simple iterative threshold method, we found that a much less aggressive filter of $10 \mathrm{~Hz}$ could be used to obtain optimal results. Because filtering can distort the signals of interest, a less aggressive filter in general produces less distortion of the signal and is more desirable for estimating response onset latency. With a higher cut-off at $10 \mathrm{~Hz}$, we were able to preserve signals within a frequency band that is important in defining not only P3, but other earlier and faster components as well, such as M 100. F or the particular components of evoked responses studied in this paper, the changes in the roll-off of the filter did not make a large difference in either the number of events detected or the quality of detection. We could not easily compare the roll-off used here with those used by some of these earlier studies because such information was not readily available. 

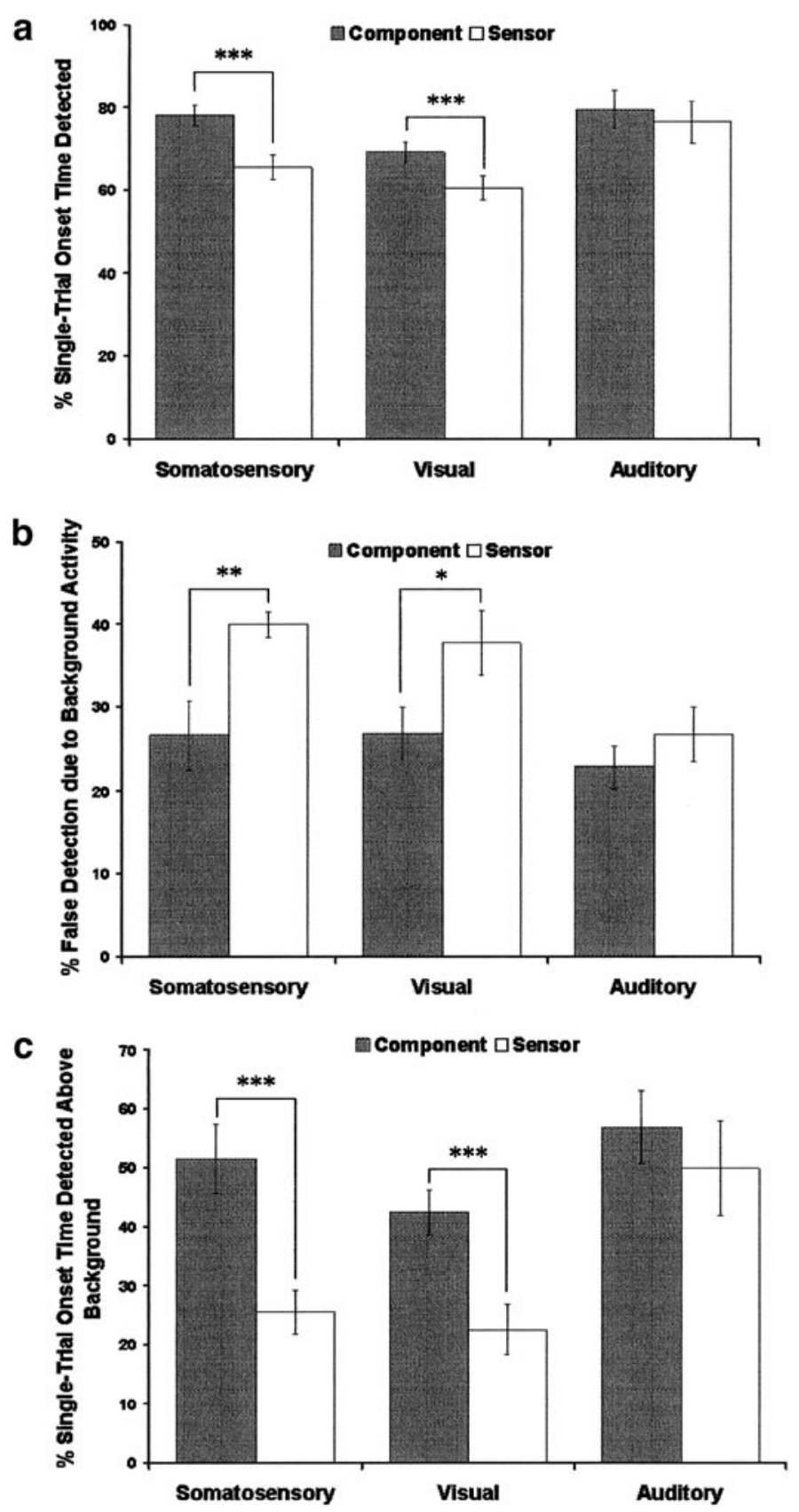

FIG. 9. Comparison of detection rates for components and sensors across all modalities for (a) onset detection in the response window, (b) false detection due to background activity in the control window, (c) onset detection above background activity (No. of detections in the response window - No. of detections in the control window). ${ }^{* * *} \mathrm{P}<0.005$; **P $<0.025$; $* \mathrm{P}<0.05$.

\section{Comparison with ICA-Aided Single-T rial EEG Data Analysis}

Applying Infomax ICA (Bell and Sejnowski, 1995b), another ICA algorithm, to EEG data, J ung et al . (1999) generated images of single-trial responses (ERP images) for the separated components. By re-sorting single-trial data using reaction times, J ung et al. (2001) showed that some ICA components are meaningful with respect to the behavioral output (reaction times). They applied the well-known Woody's template matching method to P3 from the ICA components during a visual attention task. Because the shift used in matching single-trial response to the average ERP was not displayed over the single-trial ERP image (comparable to our MEG image), it is difficult to compare the latency estimation with the response onset time results presented here.

Additional differences between this MEG study and the EEG study are: (1) we explicitly measured singletrial re sponse onset times while the latency in the EEG study was given by the shift that produced the best match to the average ERP; (2) our MEG images did not contain any vertical smearing via application of a filter across trials used in the EEG study, therefore offering better preservation of true trial-to-trial variability in evoked responses; (3) the ICA components derived from MEG data were localized to specific brain regions while dipole localization for the $\mathrm{P} 3$ components was not performed in the EEG study; and (4) we systematically applied the response onset time detection to neuronal sources across three major sensory modalities and across multiple subjects, while in the EEG study Woody's method for latency estimation was applied to the visual P3 component alone.

\section{The Independence Assumption}

For a thorough discussion of the independence assumption as it pertains to MEG, see Vigário et al. (2000). For more detailed discussion of assumptions made by SOBI within the context of MEG, see Tang et al. (2002). To help the reader evaluate the method used here, we briefly discuss what the independence assumption means in SOBI. Because ICA methods in general make an independence assumption concerning the underlying sources, it would seem impossible that such an assumption could ever be met. However, instead of assuming a zero correlation, SOBI algorithm works by minimizing the total correlations among putative sources at multiple time delays. It is possible to minimize the total correlation while allowing a few relatively larger correlations between sources, among which higher correlations are expected. Therefore, SOBI's particular method of maximizing independence is not necessarily incompatible with our expectation of a large correlation at a particular time delay be tween two sources sharing common inputs.

Second, because correlation is not a binary quantity, neither is violation of the independence assumption. The important question is not whether the assumption is violated but whether the assumption is sufficiently violated such that the estimated neuronal sources by SOBI are no longer meaningful. Therefore, whether an assumption is sufficiently violated becomes an empirical question. We have shown that SOBI was able to separate visual components that clearly correspond to neuronal responses from early and later visual processing stages that are correlated due to common input (Tang et al., 2000b). In addition, SOBI-separated components show characteristic visual, auditory, and somatosensory evoked responses and have characteristic equivalent dipole 
localizations (Tang \& al., 2002). The neurophysiological and neuroanatomical interpretability of the SOBI components gives us confidence that the method is reasonably robust even if the assumptions are not completely met. Application of several ICA algorithms to phantom data provide further support for the effectiveness and high performance in source decomposition by ICA methods (Cao et al., 2000).

\section{ACKNO WLEDGMENTS}

This work was supported by DARPA, the MIND Institute, an NSF Career Award (97-02-311), and gifts from George Cowan of SFI and from the NEC Research Institute. We thank Ole J ensen for tips on packaging the ICA separated components for the Neuromag software, Mike Weisend for granting us access to his data, and Robert Christner for technical support. We thank Claudia Tesche, Cheryl Aine, and Roland Lee for their comments and discussion.

\section{REFERENCES}

Bell, A. J ., and Sejnowski, T. J . 1995a. Blind separation and blind deconvolution: An Informationtheoretic approach. In Proceedings IEEE Conference on Acoustics, Speech, and Signal Processing, Detroit, pp. 3415-3418.

Bell, A. J ., and Sejnowski, T. J . 1995b. An information-maximization approach to blind separation and blind deconvolution. Neural Comput. 7: 1129-1159.

Belouchrani, A., Meraim, K. A., Cardoso, J.-F., and Moulines, E. 1993. Second-order blind separation of correlated sources. In Proceedings International Conference on Digital Signal Processing, Cyprus, pp. 346-351.

Brenner, D., Lipton, J ., Kaufman, L., and Williamson, S. J . 1978. Somatically evoked magnetic fields of the human brain. Science 199: 81- 83.

Brenner, D., Williamson, S. J ., and Kaufman, L. 1975. Visually evoked magnetic fields of the human brain. Science 190: 480-482.

Cao, J . T., Murata, N., Amari, S., Cichocki, A., Takeda, T., Endo, H., and Harada, N. 2000. Single-trial magnetoencephalographic data decomposition and localization based on independent component analysis approach. IEICE Trans. Fundam. Electron. Commun. Comput. Sci. E 83A: 1757-1766.

Cardoso, J .-F. 1994. On the performance of orthogonal source separation algorithms. In European Signal Processing Conference, Edinburgh, pp. 776-779.

Cardoso, J .-F., and Souloumiac, A. 1993. Blind beamforming for non Gaussian signals. IEEE Proc. F 140: 362-370.

Chung, G., Tucker, D., West, P., Potts, G., Liotti, M., Luu, P., and Hartry, A. 1996. Emotional expectancy: Brain electrical activity associated with an emotional bias in interpreting life events. Psychophysiology 33: 218-233.

Fein, G., and Turetsky, B. 1989. P300 latency variability in normal elderly: Effects of paradigm and measurement technique. Electroencephalogr. Clin. Neurophysiol. 72: 384-394.

Ford, J ., White, P., Lim, K., and Pfefferbaum, A. 1994. Schizophrenics have fewer and smaller P300s: A single-trial analysis. Biol. Psychiatry 35: 96-103.

Hari, R. 1994. Human cortical functions revealed by magnetoencephalography. Prog. Brain Res. 100: 163-168.

Hari, R., Aittoniemi, K., J arvinen, M.-L., and Varpula, T. 1980. Auditory evoked transient and sustained magnetic fields of the human brain. Exp. Brain Res. 40: 237-240.
Hari, R., and Forss, N. 1999. Magnetoencephalography in the study of human somatosensory cortical processing. Philos. Trans. R. Soc. London B 354: 1145-1154.

Huang, M., Leahy, R. M., Mosher, J . C., and Spencer, M. E. 1998a. Comparing the source localization accuracy of EEG and MEG for different head modeling techniques using a human skull phantom. Int. J . Psychophysiol. 30: 200.

Huang, M. X., Aine, C. J ., Supek, S., Best, E., Ranken, D., and Flynn, E. R. 1998b. Multi-start downhill simplex-method for spatiotemporal source localization in magnetoencephalography. Electroencephalogr. Clin. Neurophysiol. 108: 32-44.

Hyvärinen, A., and Oja, E. 1997. A fast fixed-point algorithm for independent component analysis. Neural Comput. 9.

I oannides, A. 1998. Fundamental macro-properties of brain activities revealed by magnetoencephalography (MEG). ICONIP: International Conference on Neural I nformation Processing 1: 133-138.

Ioannides, A. 2001. Real time human brain function: Observations and inferences from single trial analysis of magnetoencephalographic signals. Clin. Electroencephalogr. 32: 98-111.

I oannides, A., Liu, L., Kwapien, J ., Drozdz, S., and Streit, M. 2000a. Coupling of regional activations in a human brain during an object and face affect recognition task. Hum. Brain Mapp. 11: 77-92.

I oannides, A. A., Liu, L. C., Theofilou, D., Dammers, J ., Burne, T., Ambler, T., and Rose, S. 2000b. Real time processing of affective and cognitive stimuli in the human brain extracted from MEG signals. Brain Topogr. 13: 11-19.

I oannides, A. A., Liu, M. J ., Liu, L. C., Bamidis, P. D., Hellstrand, E., and Stephan, K. M. 1995. Magnetic-field tomography of cortical and deep processes: Examples of real-time mapping of averaged and single trial MEG signals. Int. J . Psychophysiol. 20: 161-175.

J askowski, P., and Verleger, R. 1999. Amplitudes and latencies of singletrial ERP's estimated by a maximum-likelihood method. IEEE Trans. Biomed. Eng. 46: 987-993.

J askowski, P., and Verleger, R. 2000. An evaluation of methods for single-trial estimation of P3 latency. Psychophysiol. 37: 153-162.

J in, Y., Potkin, S., Patterson, J ., Sandman, C., Hetrick, W., and Bunney, W. 1997. Effects of P50 temporal variability on sensory gating in schizophrenia. Psychiatry Res. 70: 71- 81.

J ung, T., Makeig, S., Westerfield, M., Townsend, J ., Courchesne, E., and Sejnowski, T. 2001. Analysis and visualization of single-trial event-related potentials. Hum. Brain Mapp. 14: 166-185.

J ung, T.-P., Makeig, S., Westerfield, M., Townsend, J ., Courchesne, E., and Sejnowski, T. J . 1999. Analyzing and visualizing singletrial event-related potentials. In Advances in Neural Information Processing Systems, Vol. 11, pp. 118-124. MIT Press, Cambridge, MA.

Karhu, J ., and Tesche, C. D. 1999. Simultaneous early processing of sensory input in human primary (SI) and secondary (SII) somatosensory cortices. J . Neurophysiol. 81: 2017-2025.

Kyuhou, S., and Okada, Y. 1993. Detection of magnetic evoked fields associated with synchronous population activities in the transverse CA1 slice of the guinea-pig. J . Neurophysiol. 70: 2665-2668.

Liu, B. C., I oannides, A. A., and Streit, M. 1999. Single trial analysis of neurophysiological correlates of the recognition of complex objects and facial expressions of emotion. Brain Topogr. 11: 291-303.

Liu, L. C., and I oannides, A. A. 1996. A correlation study of averaged and single trial MEG signals: The average describes multiple histories each in a different set of single trials. Brain Topogr. 8: 385-396.

Mackert, B., Wübbeler, G., Leistner, S., Trahms, L., and Curio, G. 2001. Non-invasive single-trial monitoring of human movementrelated brain activation based on DC-magnetoencephalography. NeuroReport 12: 1689-1692. 
Makeig, S., Westerfield, M., J ung, T.-P., Covington, J ., Townsend, J ., Sejnwoski, T. J ., and Courchesne, E. 1999. Functionally independent components of the late positive event-related potential during visual spatial attention. J . Neurosci. 19: 2665-2680.

Michalewski, H., Prasher, D., and Starr, A. 1986. Latency variability and temporal interrelationships of the auditory event-related potentials ( 1, p2, n2, and p3) in normal subjects. Electroencephalogr. Clin. Neurophysiol. 65: 59-71.

Okada, Y., and Xu, C. 1996. Singleepoch neuromagnetic signals during epileptiform activities in guinea pig longitudinal CA3 slices. Neurosci. Lett. 211: 155-158.

Pfefferbaum, A., Christensen, C., Ford, J., and Kopell, B. 1986. Apparent response incompatibility effects on P3 latency depend on the task. Electroencephalogr. Clin. Neurophysiol. 64: 424-437.

Pfefferbaum, A., Ford, J ., Wenegrat, B., Roth, W., and Kopell, B. 1984. Clinical-application of the P3 component of event-related potentials. 1. Normal aging. Electroencephalogr. Clin. Neurophysiol. 59: 85-103.

Pham, D., Mocks, J ., Kohler, W., and Gasser, T. 1987. Variable latencies of noisy signals: Estimation and testing in brain potential data. Biometrika 74: 525.

Roberts, T., Ferrari, P., Stufflebeam, S., and Poeppel, D. 2000. Latency of the auditory evoked neuromagnetic field components: Stimulus dependence and insights toward perception. J . Clin. Neurophysiol. 17: 114-129.

Romani, G., Williamson, S., and Kaufman, L. 1982. Tonotopic organization of the human auditory cortex. Science 216: 1339-1340.

Smulders, F., Kenemans, J., and Kok, A. 1994. A comparison of different methods for estimating single-trial P300 latencies. Electroencephalogr. Clin. Neurophysiol. 92: 107-114.

Stufflebeam, S., Poeppel, D., and Roberts, T. 2000. Temporal encoding in auditory evoked neuromagnetic fiel ds: Stochastic resonance. NeuroReport 11: 4081- 4085.

Supek, S., Aine, C. J ., Ranken, D., Best, E., Flynn, E. R., and Wood, C. C. 1999. Single vs. paired visual stimulation: Superposition of early neuromagnetic responses and retinotopy in extrastriate cortex in humans. Brain Res. 830: 43-55.

Tang, A. C., Pearlmutter, B. A., Malaszenko, N. A., Phung, D. B., and Reeb, B. C. 2002. Localization of independent components of mag- netoencephalography in cognitive tasks. Neural Comput. 14: 1827-1858.

Tang, A. C., Pearlmutter, B. A., Zibulevsky, M., and Carter, S. A. 2000a. Blind separation of multichannel neuromagnetic responses. Neurocomputing 32-33: 1115-1120.

Tang, A. C., Pearlmutter, B. A., Zibulevsky, M., Hely, T. A., and Weisend, M. P. 2000b. An MEG study of response latency and variability in the human visual system during a visual-motor integration task. In Advances in Neural Information Processing Systems, Vol. 12, pp. 185-191. MIT Press, Cambridge, MA.

Vigário, R., J ousmäki, V., Hämäläinen, M., Hari, R., and Oja, E. 1998. Independent component analysis for identification of artifacts in magnetoencephalographic recordings. In Advances in Neural Information Processing Systems, Vol. 10. MIT Press, Cambridge, MA.

Vigário, R., Särelä, J ., J ousmäki, V., Hämäläinen, M., and Oja, E. 2000. Independent component approach to the analysis of EEG and MEG recordings. IEEE Trans. Biomed. Eng. 47: 589-593.

Vigário, R., Särelä, J ., J ousmäki, V., and Oja, E. 1999. Independent component analysis in decomposition of auditory and somatosensory evoked fields. In Proceedings First International Conference on Independent Component Analysis and Blind Source Separation (ICA'99), Aussois, France, pp. 167-172.

Williamson, S., Kaufman, L., Curtis, S., Lu, Z., Michel, C., and Wang, J . 1996. Neural substrates of working memories are revealed magnetically by the local suppression of al pha rhythm. Electroencephalogr. Clin. Neurophysiol. Suppl. 47: 163-180.

Woody, C. 1967. Characterization of an adaptive filter for the analysis of variable latency neuroelectric signals. Med. Biol. Eng. 5: 539.

Wübbeler, G., Ziehe, A., Mackert, B.-M., Müller, K.-R., Trahms, L., and Curio, G. 2000. Independent component analysis of non-invasively recorded cortical magnetic DC-fields in humans. IEEE Trans. Biomed. Eng. 47: 594-599.

Ziehe, A., Müller, K.-R., Nolte, G., Mackert, B.-M., and Curio, G. 2000. Artifact reduction in magnetoneurography based on timedelayed second order correlations. IEEE Trans. Biomed. Eng. 47: 75- 87. 